\title{
Editorial
}

\section{Nanocomposites 2013}

\author{
Hongmei Luo, ${ }^{1}$ John Zhanhu Guo, ${ }^{2}$ Menka Jain, ${ }^{3}$ Ping Xu, ${ }^{4,5}$ and Guifu Zou ${ }^{6}$ \\ ${ }^{1}$ Department of Chemical Engineering, New Mexico State University, Las Cruces, NM 88003, USA \\ ${ }^{2}$ Dan F. Smith Department of Chemical Engineering, Lamar University, Beaumont, TX 77710, USA \\ ${ }^{3}$ Department of Physics, University of Connecticut, Storrs, CT 06269, USA \\ ${ }^{4}$ Department of Chemistry, Harbin Institute of Technology, Harbin 150001, China \\ ${ }^{5}$ Chemistry Division, Los Alamos National Laboratory, Los Alamos, NM 87545, USA \\ ${ }^{6}$ School of Energy, Soochow University, Suzhou 215000, China \\ Correspondence should be addressed to Hongmei Luo; hluo@nmsu.edu
}

Received 30 October 2013; Accepted 30 October 2013

Copyright (c) 2013 Hongmei Luo et al. This is an open access article distributed under the Creative Commons Attribution License, which permits unrestricted use, distribution, and reproduction in any medium, provided the original work is properly cited.

Composite materials that traditionally incorporate micronscale reinforcements in a bulk matrix offer opportunities to tailor material properties such as hardness, tensile strength, and thermal and electrical conductivity. With the advent of nanomaterials, nanocomposites are multiphase materials where one of the phases has at least one dimension of less than $100 \mathrm{~nm}$. The properties of nanocomposite materials depend not only on the properties of the building components but also on their morphologies, microstructures, and interfacial characteristics. The published works are briefly addressed as follows.

The paper from Canada reports that dispersion, microstructure, and dielectric response of polyethylene (PE)/clay nanocomposites were affected by the incorporation of anhydride modified polyethylene (PE-MA) as the compatibilizer. It was observed that the relaxation rate of the MaxwellWagner-Sillars process increased as the degree of dispersion increased. This correlation shows that broadband dielectric spectroscopy can be used as a macroscopic tool to evaluate the quality of dispersion in nanocomposite materials.

The paper from Egypt studies the rheological behavior of a different concentration of carbon nanotubes (CNTs) as additive on lithium grease under various settings of shear rate, shear stress, and apparent viscosity. The authors concluded that the optimum percentage of CNTs in the grease composite was $2 \%$ and further confirmed the importance of the correlation between rheological properties and the grease microscopic structure.
Another paper from India is about the biological synthesis of silver nanoparticles (AgNPs) using cannonball leaves. Such AgNPs showed cytotoxicity to human breast cancer cell line (MCF-7). Overall, this environment-friendly method of biological Ag nanoparticles production can potentially be used in various human contacting areas such as cosmetics, foods, and medical applications.

The other paper in this issue, also from India, is about the mechanical properties and thermal conductivity investigation of epoxy resin by dispersion multiwalled carbon nanotubes (MWCNTs) in the composites. It was found that the compressive strength, Young's modulus, and thermal conductivity of composites with MWCNTs were increased compared to those of pure epoxy. By comparison with different methods, the paper concluded that a unique method for the dispersion of MWCNTs in epoxy is the solvent dispersion technique with vacuum drying process.

Hongmei Luo John Zhanhu Guo Menka Jain Ping Xu Guifu Zou 

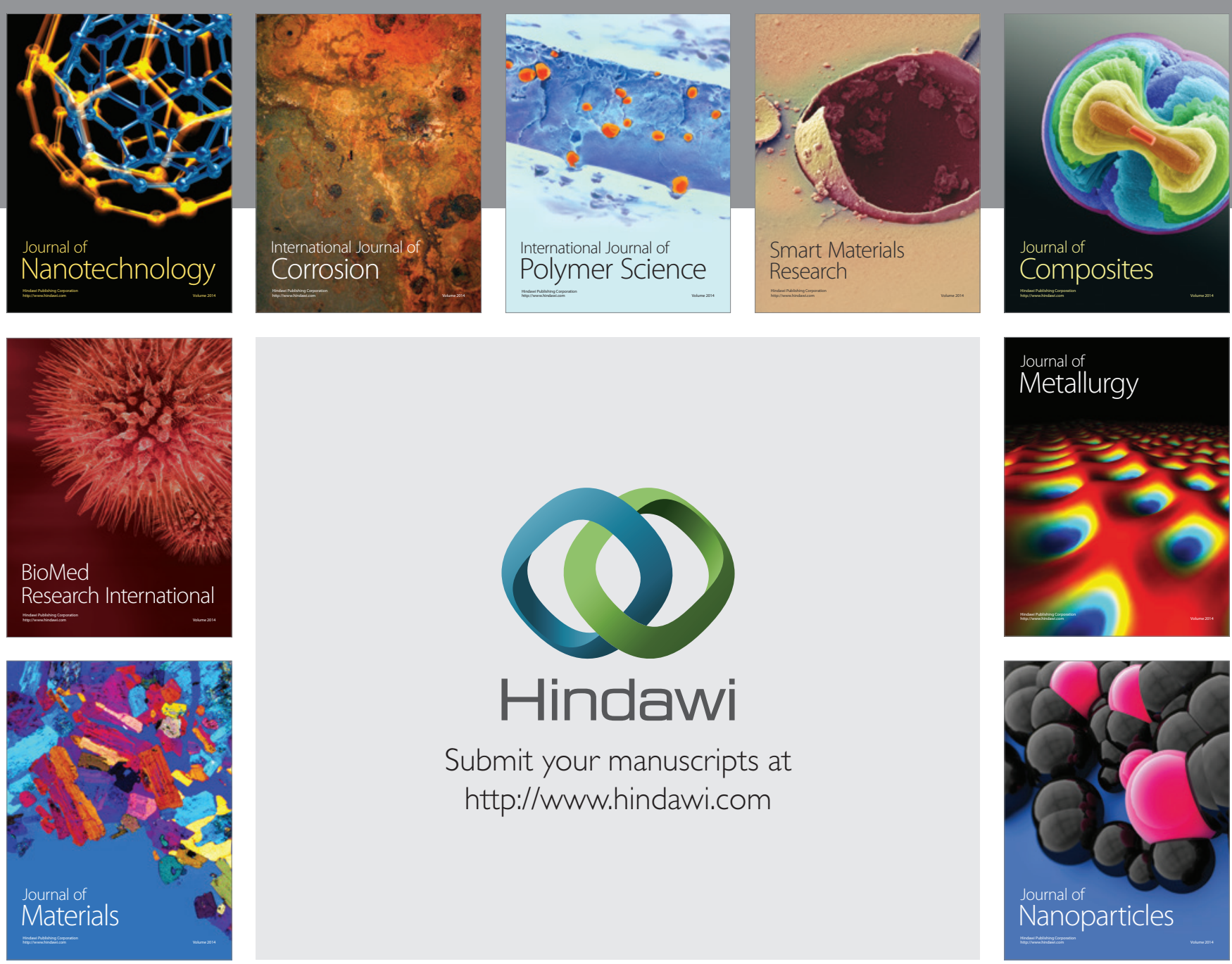

Submit your manuscripts at http://www.hindawi.com
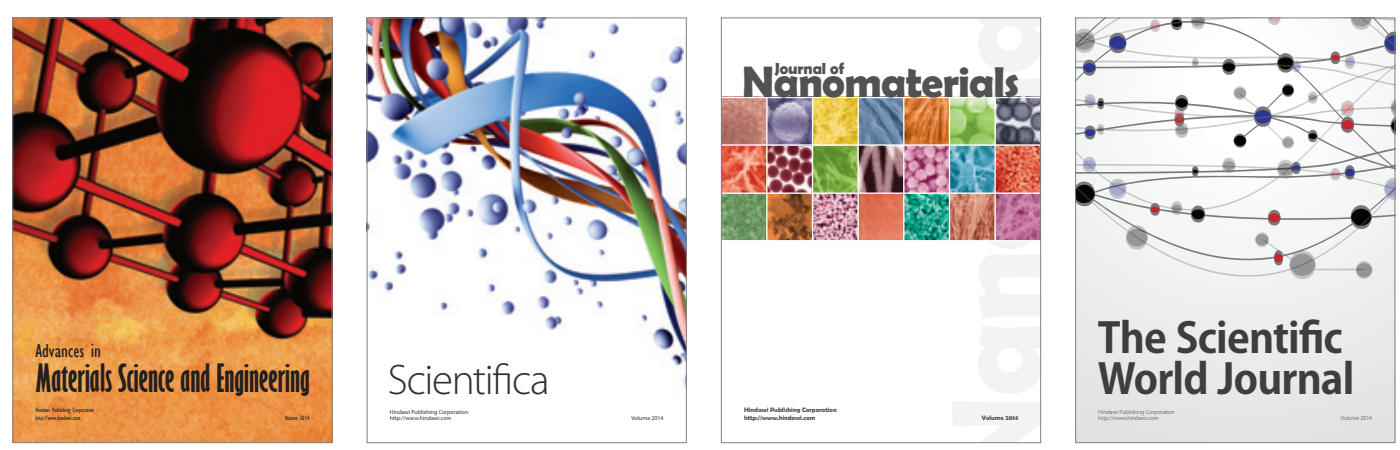

\section{The Scientific World Journal}
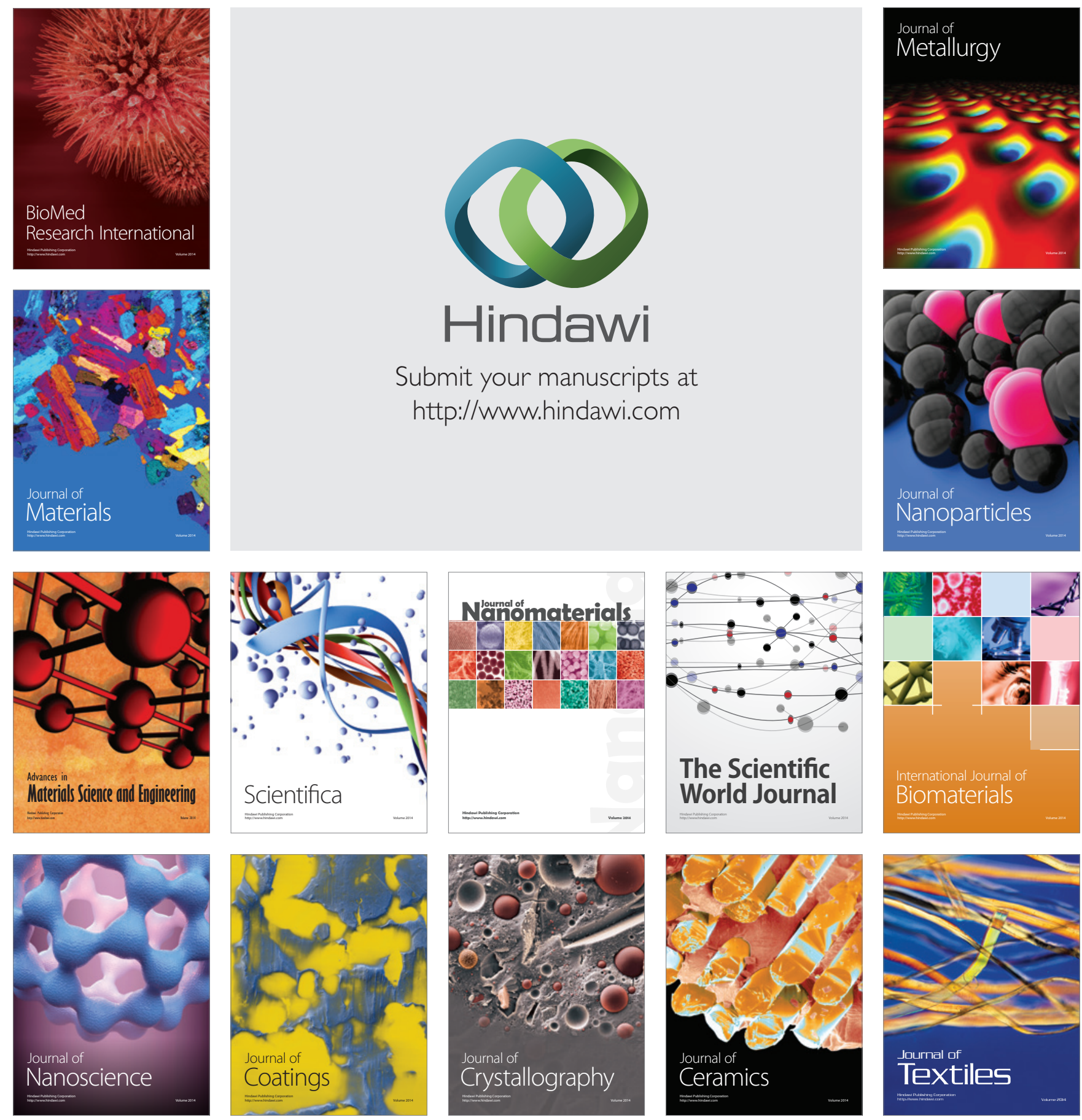